Cite this: CrystEngComm, 2013, 15, 1257

Received 2nd October 2012,

Accepted 22nd November 2012

DOI: $10.1039 / \mathrm{c} 2 \mathrm{ce} 26604 \mathrm{k}$

www.rsc.org/crystengcomm

\section{Porous tablets of crystalline calcium carbonate via sintering of amorphous nanoparticles $\dagger$}

\author{
Denis Gebauer, Xingmin Liu, Baroz Aziz, Niklas Hedin* and Zhe Zhao§*
}

\section{Introduction}

Porous calcium carbonates are used as fillers in paper, ${ }^{1}$ and are studied for applications in tooth paste ${ }^{2}$ and as a substrate for the controlled delivery of drugs. ${ }^{3}$ Nanometer sized pores can be introduced into calcium carbonates with or without assistance from organic molecules or polymers. ${ }^{4-7}$ Templatefree methods to synthesize porous calcium carbonates could have advantages over template-based ones, as template removal from internal surfaces and voids in composites of calcium carbonate and organic molecules can be complicated. Micrometer sized pores can be patterned into crystals of calcite by crystallization around micropatterns or particles. ${ }^{8,9}$ Porous powders are seldom useful in practical applications as such, and they often need to be assembled into macroscopic shapes designed for a particular application. Nandi et al. showed that macroscopic membranes could be produced by

Department of Materials and Environmental Chemistry, Arrhenius Laboratory, Stockholm University, SE-106 91 Stockholm, Sweden.

E-mail: niklas.hedin@mmk.su.se; zhezhao@kth.se.

$\dagger$ Electronic supplementary information (ESI) available. See DOI: 10.1039/ c2ce $26604 \mathrm{k}$

\$ Current address: Department of Chemistry, Physical Chemistry, University of Konstanz, Universitätsstrasse 10, D-78457 Konstanz, Germany.

$\S$ Current address: Department of Materials Science and Engineering, Royal Institute of Technology, SE-100 44, Stockholm, Sweden. sintering commercial powders of calcium carbonate at high temperatures. ${ }^{10}$ These membranes had large pores $(>500 \mathrm{~nm})$ and porosities of $30-40 \%$. Although such large pores are relevant for certain applications many applications need smaller pores.

In this study, we apply a relatively new method for sintering, often referred to as spark plasma sintering to produce tablets of calcium carbonate with small pores. It uses pulsed direct currents to heat the sample, instead of an external heat source. It has been successfully applied to sinter various types of structured ceramics and composites. The heating via pulsed currents allows unusually rapid heating, and consequently to that, densification can be achieved at lower temperatures than with traditional methods. ${ }^{11}$ This kind of pulse current processing has also been used to fuse particles of meso- and microporous silicates together without losing the porosity; ${ }^{12,13}$ studies that led us to investigate if this sintering method would be useful for porous calcium carbonates as well.

Amorphous calcium carbonate (ACC) has a $40-50 \%$ lower density than its anhydrous and crystalline forms. ${ }^{14}$ Hence, ACC often tends to form more rapidly in aqueous solutions than the crystalline forms of calcium carbonate, calcite being the thermodynamically stable form. This tendency is in agreement with the Ostwald-Volmer rule. In aqueous solutions and without additives, ACC most often transforms to 
vaterite and calcite at low temperatures, and at elevated temperatures $>40{ }^{\circ} \mathrm{C}$ also with the presence of aragonite. ${ }^{15-18}$ Precipitated ACC crystallizes in aqueous solutions if it is not rapidly isolated or otherwise stabilized, for example by subjecting the sample to low temperatures. The solubility of ACC increases with a decreasing temperature, as was established separately by Brečević and Nielsen ${ }^{19}$ and Clarkson et al. ${ }^{20}$ Even though its solubility is higher at low temperatures, RodriguesBlanco et al. ${ }^{18}$ showed that the transformation of ACC to vaterite was slower at a temperature of $7.5{ }^{\circ} \mathrm{C}$ than at $25{ }^{\circ} \mathrm{C}$. In general, the formation of amorphous compounds is promoted at high levels of super-saturation, and Brečević and Nielsen ${ }^{19}$ showed that nanoparticles of ACC precipitated at high concentrations of $\mathrm{Ca}^{2+}$ and $\mathrm{CO}_{3}{ }^{2-}$. Another way to enhance the super-saturation is by using solvents with lower dielectric constants than water and thereby lowering the solubility of the amorphous phase. Lee et al. showed that unusually stable nanoparticles of ACC precipitated in ethanol. ${ }^{21}$ The authors precipitated ACC by dissolving ammonia and $\mathrm{Ca}^{2+}$ in ethanol and subjecting the solution to $\mathrm{CO}_{2}$ gas. In a related manner, we synthesized small nanoparticles of ACC by inducing a high super-saturation of $\mathrm{Ca}^{2+}$ and $\mathrm{CO}_{3}{ }^{2-}$ in an ethanol-water solution by rapid addition of a reactant. ${ }^{22,23} \mathrm{Na}_{2} \mathrm{CO}_{3}$ was rapidly added to an ethanol-rich solution containing $\mathrm{Ca}^{2+} \cdot{ }^{23}$ The rapidly increasing supersaturation on such addition promoted the rapid nucleation of virtually monodisperse nanoparticles of ACC. The high level of super-saturation enabled a kinetic control over the precipitation of ACC, which is in agreement with the Ostwald-Volmer rule. No crystalline calcium carbonate nucleated in parallel to ACC. Here, we show that nanoparticles of ACC obtained in this manner can be used as precursors to monolithic tablets of porous and crystalline calcium carbonate by using compressive stress $(20 \mathrm{MPa})$ and a moderate temperature $\left(120{ }^{\circ} \mathrm{C}-400{ }^{\circ} \mathrm{C}\right)$, induced by pulsed direct currents in a sintering device.

\section{Experimental section}

\section{Materials}

ACC was synthesized with a modified version of the protocol presented by Gebauer et $a .^{23}$ The modification allowed us to increase the concentrations of reactants five-fold in comparison with the earlier study. Calcium chloride hexahydrate (0.52.5 mmol; Sigma Aldrich No. 21108, CAS number: 7774-34-7) was dissolved in $50 \mathrm{ml}$ of water. The aqueous solution was mixed with $2 \mathrm{dm}^{3}$ of ethanol (99.9\%; VWR No. 20820.293, CAS number: 64-17-5) in a plastic beaker made of polypropylene. An equimolar amount of sodium carbonate was dissolved in $50 \mathrm{ml}$ of water (0.5-2.5 mmol; Sigma Aldrich, CAS number: 497-19-8). This aqueous solution was quickly added to the ethanol-rich solution described above. The plastic beaker was sealed with Parafilm ${ }^{\mathrm{TM}}$. The mixture was first stirred for $\sim 30$ minutes and then left unstirred for $\sim 30$ minutes. Translucent ACC sedimented in the bottom of the plastic beaker, and the supernatant solution was carefully decanted. The sediment was centrifuged at $\sim 6000 \mathrm{~g}$ and the supernatant was decanted. The sediment was re-suspended in ethanol, centrifuged and decanted, re-suspended in acetone (VWR No. 20066.296), centrifuged, and decanted, and finally dried under conditions of dynamic vacuum. A single synthesis produced $\sim 250 \mathrm{mg}$ of ACC with a close to stoichiometric yield.

\section{Sintering}

Powders of ACC were subjected to a compressive stress of 20 $\mathrm{MPa}$ and pulsed direct currents that rapidly raised the temperature using a sintering device (Dr. Sinter 2050, Sumitomo Coal Mining Co., Tokyo, Japan). 100 mg of ACC was loaded into a steel die with an inner diameter of $8 \mathrm{~mm}$. The setup was heated to targeted temperatures in the range of 120-400 ${ }^{\circ} \mathrm{C}$ using different rates of heating $\left(20-100{ }^{\circ} \mathrm{C} \mathrm{min}^{-1}\right)$. The dwelling time at targeted temperature was three minutes and further details regarding the sintering profile are described by Zhao et $a .^{24}$

\section{Scanning electron microscopy (SEM)}

A JEOL JSM-7000F microscope was used to record SEM images in a secondary electron imaging mode. An acceleration voltage of $2.5 \mathrm{kV}$ was used. Powders of ACC were sprinkled on sample holders covered with carbon-ink and allowed to dry. A few samples were coated with a thin layer of carbon $(\sim 10 \mathrm{~nm})$.

\section{$\mathrm{X}$-ray powder diffraction (XRD)}

X-ray diffractograms were recorded on a PANalytical X'Pert Pro diffractometer using $\mathrm{Cu} \mathrm{K} \alpha_{1}(\lambda=1.5406 \AA)$ radiation and a PIXel detector. Diffractograms were acquired at $20^{\circ}<2 \theta<60^{\circ}$ with a scanning speed of $0.04^{\circ} \mathrm{s}^{-1}(\sim 17$ minutes per diffractogram). Finely ground powders of calcium carbonate were dispersed in isopropanol and spread uniformly on silicon plates. Where indicated, whole tablets were specifically oriented in custom-made putty sample holders. The powder XRD data were analyzed using the X'Pert Highscore Plus program. Two-dimensional X-ray diffraction patterns were recorded at room temperature on an Oxford Diffraction Xcalibur3 diffractometer using Mo $\mathrm{K} \alpha$ radiation $(\lambda=$ $0.71073 \AA$ ) and a Sapphire-3 CCD detector (with Gaussian absorption correction). Fractured pieces of $\sim 0.5 \mathrm{~mm}$ in diameter of the sintered tablets were exposed to the X-ray beam for five minutes, both from the surface and from the bulk of the tablets.

\section{Infrared spectroscopy}

Fourier transform infrared (FTIR) spectra were recorded on a Varian 670-IR spectrometer equipped with an attenuated total reflection detector (Goldengate by Specac). Spectra were recorded on finely ground powders at a spectral resolution of $4 \mathrm{~cm}^{-1}$.

\section{$\mathbf{N}_{2}$ adsorption}

Adsorption and desorption isotherms of $\mathrm{N}_{2}$ was measured at the boiling point of $\mathrm{N}_{2}\left(-196{ }^{\circ} \mathrm{C}\right)$ with either a Micromeritics Gemini 2375 device, or a Micromeritics ASAP2020 device. Before experimentation, occluded molecules were removed, either by subjecting the samples to a stream of dry $\mathrm{N}_{2}$ for at $>20$ hours at a temperature of $300{ }^{\circ} \mathrm{C}$ in a Micromeritics FlowPrep 60 system, or by subjecting them to conditions of dynamic vacuum and a temperature of $150{ }^{\circ} \mathrm{C}$ for six hours. 
Specific surface areas were evaluated in the model of Brunauer, Emmett and Teller (BET) using the adsorbed amount of $\mathrm{N}_{2}$ at relative pressures of $0.06-0.3$.

\section{Result and discussion}

\section{Nanoparticles of amorphous calcium carbonate}

We extended the protocol of Gebauer et $a .^{23}$ to be able to synthesize large enough quantities of ACC. ACC was precipitated as nanoparticles from ethanol-rich mixtures of ethanol and water (5.7 vol\% water) by reacting equimolar quantities of $\mathrm{CaCl}_{2}$ and $\mathrm{Na}_{2} \mathrm{CO}_{3}$. Sediments of aggregated nanoparticles of ACC were separated from the reaction mixtures before they started to transform into their crystalline polymorphs. Each synthetic batch was analysed to assure the amorphous nature by FTIR spectroscopy (a typical FTIR spectrum is presented in the ESI, $\dagger$ Fig. S1). In about one out of ten batches there were traces of aragonite, which were discarded. For each batch $\sim 250 \mathrm{mg}$ of ACC were produced with close to a stoichiometric yield, and we regard further scale-up to be straightforward.

The nanoscopic character of the precipitated ACC was analyzed by SEM and by measuring the specific surface area. Fig. 1 shows four SEM images of small particles of ACC. Uncoated particles of ACC are displayed in Fig. $1 \mathrm{a}$ and $\mathrm{b}$ and the average particle size for these particles was $\sim 10 \mathrm{~nm}$. As SEM images of ACC are prone to artifacts, carbon coated particles of ACC were also imaged. Fig. 1c and d display SEM images of ACC particles coated with carbon and the particles size was $\sim 30 \mathrm{~nm}$, consistent with a carbon layer with a thickness of $\sim 10 \mathrm{~nm}$. The SEM micrographs indicate a narrow particle size distribution. These SEM images are highly similar to those observed by Gebauer et al. ${ }^{23}$ who also established that the particle distribution was narrow and that the average size
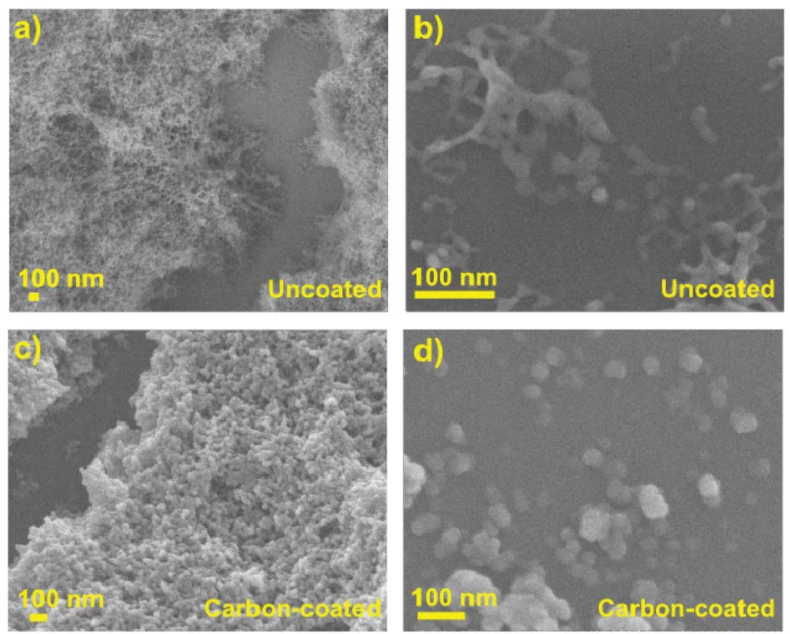

Fig. 1 Scanning electron micrographs of the precursor powder. Uncoated powders of amorphous calcium carbonate (ACC) are shown in (a) and (b). ACC powders coated with a thin layer of carbon are shown in (c) and (d). Uncoated ACC tended to melt due to heating by the electron beam, but coated ACC was stable. of ACC was $\sim 11 \mathrm{~nm}$ when synthesized under similar conditions by using Small Angle X-ray Scattering (SAXS).

Analyzes of nitrogen adsorption isotherms gave specific surface areas of $\sim 140-160 \mathrm{~m}^{2} \mathrm{~g}^{-1}$ for the precursor nanoparticles of ACC. A representative nitrogen adsorption isotherm is presented in the ESI $\dagger$ (Fig. S2). The specific surface area was invariant to the rather limited concentration range of $\mathrm{Ca}^{2+}$ used for the precipitation of ACC. These specific surface areas compare to those observed by Cai et al. They determined the specific surface area to be $\sim 120 \mathrm{~m}^{2} \mathrm{~g}^{-1}$ for particles of ACC stabilized by polyacrylic acid. ${ }^{25-27}$ These high specific surface areas can be contrasted with lower specific surface areas observed by others. Kojima et $a .^{28}$ determined the surface area to be $65 \mathrm{~m}^{2} \mathrm{~g}^{-1}$ for a partly dehydrated version of ACC with the composition of $\mathrm{CaCO}_{3} \cdot 0.5 \mathrm{H}_{2} \mathrm{O}$. Radha et al. ${ }^{29}$ determined specific surface areas for synthetic and biogenic particles of ACC to be $9-42 \mathrm{~m}^{2} \mathrm{~g}^{-1}$. Note that the specific surface areas for ACC can easily vary one order of magnitude owing to differences in particle size, and high super-saturation levels of $\mathrm{Ca}^{2+}$ and $\mathrm{CO}_{3}{ }^{2-}$ promote the formation of small nanoparticles of ACC with a high specific surface area.

\section{Sintering of nanoparticles of ACC - effect of temperature}

Transformation of amorphous nanoparticles into crystalline particles and fusing of particles into macroscopic bodies are expected to be temperature dependent. Powders of ACC were sintered at temperatures of $120{ }^{\circ} \mathrm{C}-400{ }^{\circ} \mathrm{C}$. The sintering took place under compressive stress and a temperature that was rapidly increased to the set temperature. The rapid temperature increase was assured by applying pulsed direct currents throughout the steel die used, which was used to hold the powder under compressive stress. The tablets that were sintered at $250{ }^{\circ} \mathrm{C}-400{ }^{\circ} \mathrm{C}$ were stronger than those that were sintered at lower temperatures. Fig. 2 displays four items: (a) a
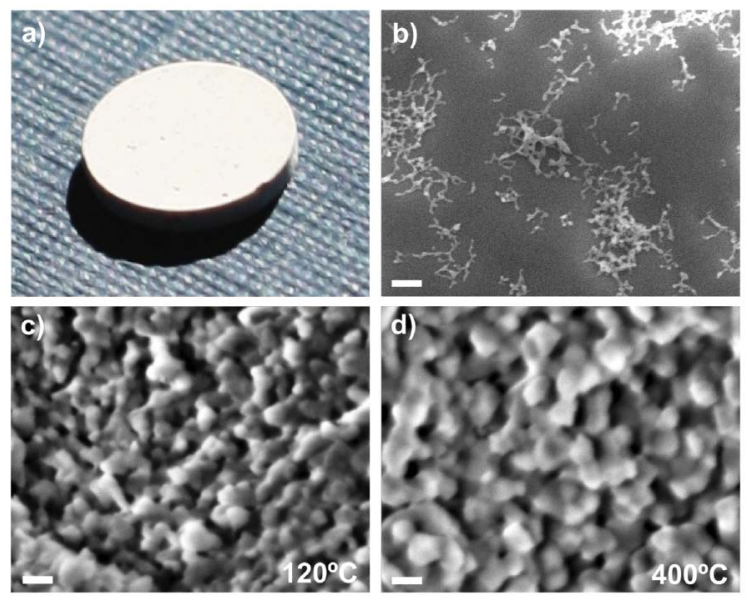

Fig. 2 Photograph of a monolithic tablet (with a diameter of $8 \mathrm{~mm}$ and a thickness of $1 \mathrm{~mm}$ ) of mesoporous calcium carbonate (a). Scanning electron microscopy (SEM) image of neat precursor nanoparticles of amorphous calcium carbonate (b); note that the particles tend to melt due to heating by the electron beam. SEM images of fracture edges of porous and monolithic tablets sintered at $120^{\circ} \mathrm{C}$ (c) and $400{ }^{\circ} \mathrm{C}(\mathrm{d})$. Scale bars: $100 \mathrm{~nm}$. The heating rate applied was $50{ }^{\circ} \mathrm{C} \mathrm{min}^{-1}$. 
photograph of a sintered and porous tablet, (b) a scanning electron microscope (SEM) image of neat nanoparticles of ACC used as precursors for the crystalline tablet, and two representative SEM images of the fracture edges for two porous tablets that had been sintered at $120{ }^{\circ} \mathrm{C}$ (c) and $400{ }^{\circ} \mathrm{C}$ (d). Fig. $2 \mathrm{c}$ and d displays SEM images of the porous structure of the tablets, which consisted of networks of well-interconnected particles of $50-100 \mathrm{~nm}$ in size. The tablets sintered at temperatures of $120{ }^{\circ} \mathrm{C}-350{ }^{\circ} \mathrm{C}$ all had BET surface areas of $15-$ $20 \mathrm{~m}^{2} \mathrm{~g}^{-1}$ (see Table 1), values which were consistent with those expected from interconnected spheres of calcium carbonate of $\sim 50 \mathrm{~nm}$ in size (smooth non-connected spheres of aragonite with a radius of $25 \mathrm{~nm}$, would have a specific surface areas of $41 \mathrm{~m}^{2} \mathrm{~g}^{-1}$ ). The tablets prepared at high temperatures appeared to contain only somewhat smaller pores than those prepared at low temperatures ( $c f$. Fig. 2c and d). The specific surface area decreased by only a minor amount on increasing the temperature, which was consistent with the minor change in particle size observed by SEM. A set of SEM images was recorded on the sintered tablets at different levels of magnification. These are presented in the ESI $\dagger$ (Fig. S3-S6). Note, Xu et $a l .{ }^{30}$ showed that films of ACC transformed to calcite after heating with no pressure applied. The overall consistency observed between the specific surface areas and the particle sizes established by SEM indicated that most, if not all, pores of the tablets were accessible to the adsorbing nitrogen gas. A reduced surface area, compared with the others, was determined for tablets sintered at $400{ }^{\circ} \mathrm{C}$, which indicated a start of pore collapse within the network.

Semi-quantitative analysis of X-ray diffractograms ${ }^{31}$ were applied to determine the average compositions of aragonite, vaterite, and calcite. The reflections were broad, indicating crystallites of nanometer size, which is consistent with the sizes estimated from SEM images and specific surface areas determined by adsorption. The surface areas reduced 5-10 fold on sintering, which was consistent with 3-5 times larger nanoparticles and a doubling of the density of the calcium carbonate. A density of $1.49 \mathrm{~g} \mathrm{~cm}^{-3}$, was reported for ACC by Bolze et $a l^{32}$ by using SAXS, and largely confirmed by Cölfen and Völkel ${ }^{14}$ in a detailed study using analytical ultracentrifugation. The density of aragonite is approximately twice that of ACC, $2.93 \mathrm{~g} \mathrm{~cm}^{-3} \cdot{ }^{14}$ Fig. 3 shows powder X-ray diffractograms of ground powders of sintered tablets of calcium carbonate. The patterns correspond to those of aragonite with a minor fraction of vaterite for tablets sintered at temperatures of $120-350{ }^{\circ} \mathrm{C}$, and to that of calcite for tablets sintered at 400 ${ }^{\circ} \mathrm{C}$. The broad reflections in the diffractograms indicated that the tablets consisted of nanocrystals. A finding that was consistent with the dimensions observed in the SEM images and the relatively large specific surface areas determined from nitrogen adsorption data. X-ray diffractograms that were recorded on intact tablets indicated that minor amounts of calcite formed on the surface of the tablets, especially in the outer rim (lateral surface) (Fig. S7, ESI $\dagger$ ). A two-dimensional diffractogram was recorded on a fractured piece of the bulk of a tablet sintered at a temperature of $300{ }^{\circ} \mathrm{C}$. Ring patterns from aragonite are visible and no obvious mutual alignment or orientation are detected (Fig. S8, ESI $\dagger$ ). It appears as the crystallites are randomly distributed within the tablets.

Although aragonite is the high-temperature-pressure polymorph of calcium carbonate, the macroscopic phase of aragonite becomes thermodynamically stable at much higher temperatures and pressures than those applied during the sintering process in this study. ${ }^{33}$ It is well-established that aragonite primarily forms in aqueous solutions at elevated temperatures and ambient pressure; ${ }^{34,35}$ however, aragonite does not form under thermodynamic control. Under these conditions the solubilities of calcite, aragonite and vaterite decrease with increasing temperature, but calcite remains less soluble than aragonite, which in turn remains less soluble than vaterite, as Kawano et al. ${ }^{34}$ showed in their summarized literature reports on the temperature dependency of the activity-based solubility products of the different polymorphs of calcium carbonate (in between $0{ }^{\circ} \mathrm{C}$ and $100{ }^{\circ} \mathrm{C}$ ). Note that the solubility product, $K_{\mathrm{sp}}$, directly reflects thermodynamic stability as it is related to the free enthalpy change as $\Delta G=$ $-R T \ln \left[1 / K_{\mathrm{sp}}\right] . \Delta G$ is the change in free enthalpy upon formation of the solid in water. This trend is in accordance with the phase stability within the solid-state as reported by Jamieson et $a .^{33}$ and hence, crystallization of the ACC on sintering occurred under kinetic control as it formed mainly aragonite with a minority fraction of vaterite, instead of calcite. Yoshino et $a l^{36}$ recently studied pressure-induced crystallization of ACC at room temperature with different amounts of water. They observed crystals of vaterite and calcite when subjecting ACC to pressures $>240 \mathrm{MPa}$ for ACC with 21 wt $\%$ water, and at somewhat higher pressures when the ACC contained less water. These pressures were significantly larger than the $20 \mathrm{MPa}$ which we used. Calcite is the thermodynamically stable modification of calcium carbonate under the conditions applied. This strongly suggests that, in fact, temperature exerts the kinetic control over aragonite formation, and this trend apparently holds true for different pathways, in solution and in the solid state. That aragonite

Table 1 Compositions and specific $\mathrm{N}_{2}$-BET surface areas for tablets sintered at different temperatures

Sintering temperature $\left[{ }^{\circ} \mathrm{C}\right] \quad$ Fraction of vaterite [\%] Fraction of aragonite [\%] Fraction of calcite [\%] Specific $\mathrm{N}_{2}$-BET surface area $\left[\mathrm{m}^{2} \mathrm{~g}^{-1}\right.$ ]

19

14

13

15

11

13

0
81

86

87

85

89

87

13
20.2

15.3

16.7

13.5

19.5

16.5

10.7 


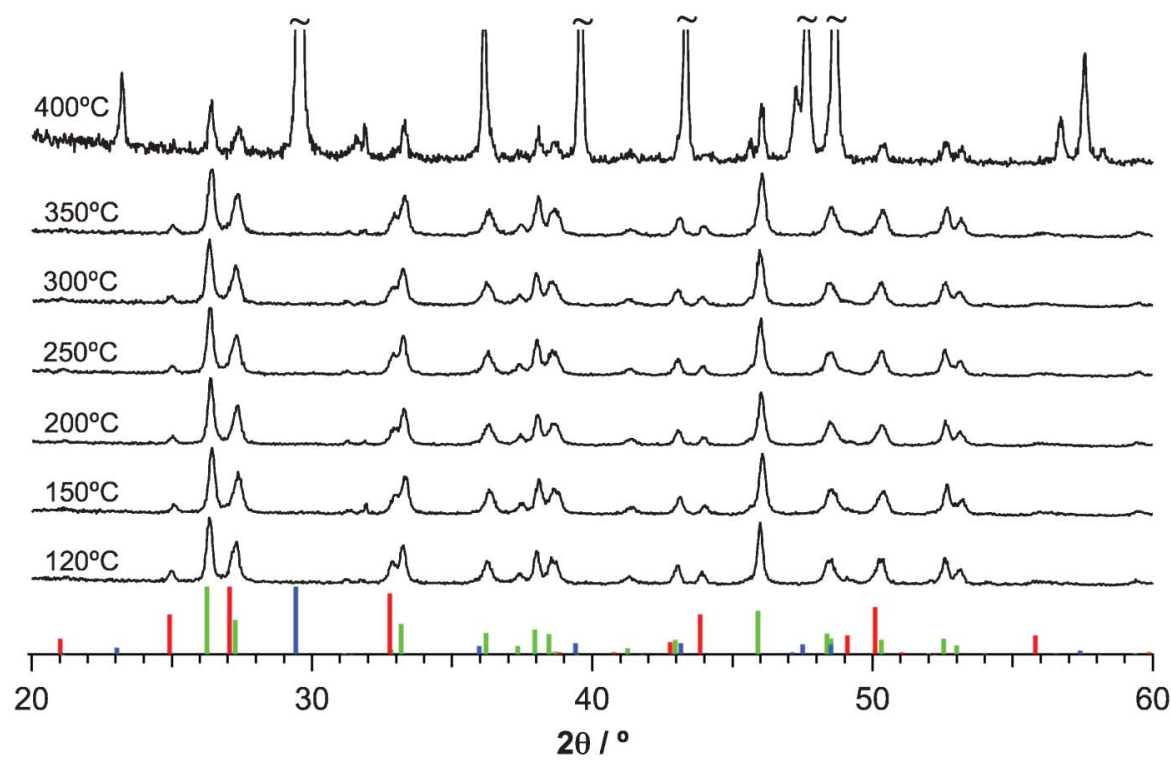

Fig. 3 Powder X-ray diffractograms of samples sintered at temperatures of $120^{\circ} \mathrm{C}-400{ }^{\circ} \mathrm{C}$ normalized to the most intense reflection for aragonite at $\sim 26^{\circ}$. Database (ICDD) diffraction patterns are shown at the bottom: calcite (blue), vaterite (red), and aragonite (green).

is the stable high-pressure polymorph of calcium carbonate (it is more dense than calcite) was not relevant at the pressures used in this study, as was apparent from that calcite formed at $400{ }^{\circ} \mathrm{C}$, which in turn can be rationalized as a solid-solid phase transition. At ambient pressures, the solid-solid phase transitions occur at $\sim 360{ }^{\circ} \mathrm{C}-500{ }^{\circ} \mathrm{C}$ for vaterite to calcite ${ }^{37-39}$ and at $\sim 400{ }^{\circ} \mathrm{C}-500{ }^{\circ} \mathrm{C}$ for aragonite to calcite. ${ }^{38,40}$ Peric et $a .^{38}$ showed that structural water (the ACC precursor particles are $\mathrm{CaCO}_{3} \cdot \mathrm{H}_{2} \mathrm{O}$, Fig. S9, ESI $\dagger$ ) accelerated the kinetics of transformation, and Baitalow et $a .^{37}$ showed that the presence of ACC lowered the temperatures for the solid-solid phase transition. It is reasonable to assume that these transition temperatures were not significantly affected by the moderate compressive pressure (20 MPa) used here. Most likely, the ACC transformed first to aragonite and vaterite, and then successively, and rapidly, to calcite at $400{ }^{\circ} \mathrm{C}$. Speculatively, this could be related to the fact that ACC mostly transforms to vaterite and aragonite at elevated temperatures in aqueous solutions. ${ }^{15-18}$ We would have expected a different morphology of the microstructure within the tablets, if ACC had transformed directly to calcite. We infer that the formation of calcite at temperatures $>400{ }^{\circ} \mathrm{C}$ was due to thermodynamic control, while the crystallization of ACC into aragonite and vaterite, at temperatures of $<400{ }^{\circ} \mathrm{C}$ was due to kinetic control.

We emphasize that under the sintering conditions applied in this study, phase transitions of ACC to crystalline forms are expected to be, or closely related to, solid-(solid)-state transitions owing to the elevated temperatures and the water free atmosphere applied. While it is known that the conditions for the crystallization can influence the type of pathways traveled, i.e. re-dissolution-crystallization or solid-solid state transformations, ${ }^{30}$ the conditions applied in our study only allow for the latter type of pathway. The question whether and how the pathway of crystallization of ACC can be controlled in solution is a topical subject, which is however not directly relevant for this study; it appears that in solution, additives can play important roles, ${ }^{41}$ but it is very likely that also especially particle size of ACC, ${ }^{42}$ spatial confinement ${ }^{43}$ and other extensive and intensive variables will influence the mode of crystallization of ACC. ${ }^{22}$

\section{Sintering of nanoparticles of ACC - effect of rate of temperature change}

Another set of tablets were produced to test the effect of rate of temperature change during the sintering. Differential rates of temperature change could induce different changes in the microstructures that develop when subjecting nanoparticles of ACC to compressive stress and an enhanced temperature. We studied the effect of the rate of temperature change (20, 50, and $100{ }^{\circ} \mathrm{C} \mathrm{min}^{-1}$ ) at four temperatures that in the earlier part of the study showed aragonite-rich tablets $(200,250,300$, and $350{ }^{\circ} \mathrm{C}$ ). Semi-quantitative analyses of the X-ray diffractograms determined the relative fractions of aragonite to vaterite within the tablets. These fractions were largely invariant to both the temperature and the rate of temperature used during the sintering. Scherrer analyzes of reflection widths revealed that the crystallite were small with a size largely invariant to both heating rate and sintering temperature. The sizes given by Scherrer analysis agreed well with the particle sizes estimated by SEM. Irrespectively of the heating rate used, the sintered porous tablets consisted of mainly aragonite with a minority fraction of vaterite. Table 2 presents data on the average composition and the size of the crystallites as a function of both the temperature and the rate of temperature change used during the sintering of ACC into tablets of porous calcium carbonate. It was striking to us how robust both the relative composition and the size of crystallites of aragonite and vaterite were to both the temperature and the rate of temperature change applied during the sintering of nanoparticles of ACC. 
Table 2 Relative amount of aragonite and vaterite, and crystallite sizes for sintered bodies of calcium carbonate as determined by semi-quantative phase analysis and Scherrer analysis of XRD data

\begin{tabular}{|c|c|c|c|c|c|}
\hline $\begin{array}{l}\text { Temperature } \\
\left({ }^{\circ} \mathrm{C}\right)\end{array}$ & $\begin{array}{l}\text { Rate of temperature } \\
\text { change }\left({ }^{\circ} \mathrm{C} \text { min }^{-1}\right)\end{array}$ & $\begin{array}{l}\text { Aragonite amount } \\
\text { (at\%) }\end{array}$ & $\begin{array}{l}\text { Aragonite crystal } \\
\text { size (nm) }\end{array}$ & $\begin{array}{l}\text { Vaterite amount } \\
\text { (at\%) }\end{array}$ & $\begin{array}{l}\text { Vaterite crystal } \\
\text { size }(n m)\end{array}$ \\
\hline 200 & 20 & 84 & 31 & 16 & 42 \\
\hline 200 & 50 & 83 & 35 & 17 & 46 \\
\hline 200 & 100 & 82 & 38 & 18 & 49 \\
\hline 250 & 20 & 83 & 35 & 17 & 45 \\
\hline $250^{a}$ & 50 & 83 & 36 & 17 & 46 \\
\hline 250 & 100 & 82 & 38 & 18 & 53 \\
\hline 300 & 20 & 80 & 30 & 20 & 39 \\
\hline 300 & 50 & 80 & 31 & 20 & 43 \\
\hline 300 & 100 & 81 & 38 & 19 & 51 \\
\hline 350 & 20 & 82 & 31 & 18 & 43 \\
\hline 350 & 50 & 79 & 33 & 21 & 44 \\
\hline 350 & 100 & 83 & 39 & 17 & 50 \\
\hline
\end{tabular}

During the pulsed currents applied to heat the samples during sintering, the temperature rose rapidly. The rapid temperature rise, combined with a short holding time, limit the time for ripening and grain growth. Smaller grains are achieved, when compared with hot pressing or other slower tools for sintering. ${ }^{11}$ Still, we observed a slight increase in the size of the crystallites on an increased rate of temperature change (10-20\%).

\section{Conclusions}

Porous tablets of crystalline calcium carbonate formed by rapidly heating nanoparticles of ACC under compressive stress. The rapid heating hindered grain growth. The tablets consisted of mainly the polymorph aragonite with a minority fraction of vaterite, when the temperature applied was $<400$ ${ }^{\circ} \mathrm{C}$. At higher temperatures calcite formed. An increase in the rate of temperature change did not affect the composition, or even increase the size of crystallites much. It could be the sintering conditions that defined the tendency of aragonite formation. As we detected batches of ACC precursors that already had traces of aragonite before sintering, it could also have been the particular nanoparticles of ACC used that were prone to aragonite formation as such. These nanoparticles of ACC were precipitated from ethanol without additives, and we expect that this procedure could be scaled up further. The porous tablets had specific surface areas of $\sim 15 \mathrm{~m}^{2} \mathrm{~g}^{-1}$, and they could be useful in applications that could be sensitive to the presence of additives, or if a large fractions of aragonite would be beneficial. Macroscopic tablets of porous aragonite are not easy to synthesize by means of other synthetic strategies than that reported here.

\section{Acknowledgements}

Denis Gebauer was supported by a postdoctoral grant from the Wenner-Gren Foundations. B.A. was supported by the Institute
Excellence Centre, CODIRECT. We thank Zoltan Bacsik, German Salazar-Alvarez, and Ehsan Jalilian (Stockholm University).

\section{References}

1 G. B. Sukhorukov, D. V. Volodkin, A. M. Günther, A. I. Petrov, D. B. Shenoy and H. Möhwald, J. Mater. Chem., 2004, 14, 2073-2081.

2 Y. Suga and Y. Ogawa, US Pat., 5882631, 1999.

3 B. V. Parakhonskiy, A. Haase and R. Antolini, Angew. Chem. Int. Ed., 2012, 51, 1195-1197.

4 J. Yu, J. C. Yu, L. Zhang, X. Wang and L. Wu, Chem. Commun., 2004, 10, 2414-2415.

5 Y.-Y. Kim, K. Ganesan, P. Yang, A. N. Kulak, S. Borukhin, S. Pechook, L. Ribeiro, R. Kröger, S. J. Eichhorn, S. P. Armes, B. Pokroy and F. C. Meldrum, Nat. Mater., 2011, 10, 890-896.

6 L. A. Estroff and I. Cohen, Nat. Mater., 2011, 10, 810-811.

7 A. Xu, M. Antonietti, S.-H. Yu and H. Cölfen, Adv. Mater., 2008, 20, 1333-1338.

8 J. Aizenberg, D. A. Muller, J. L. Grazul and D. R. Hamann, Science, 2003, 299, 1205-1208.

9 C. Li and L. Qi, Angew. Chem. Int. Ed., 2008, 47, 2388-2393.

10 B. K. Nandi, R. Uppaluri and M. K. Purkait, Appl. Clay. Sci., 2008, 42, 102-110.

11 M. Nygren and Z. Shen, Solid State Sci., 2003, 5, 125-131.

12 P. O. Vasiliev, Z. J. Shen, R. P. Hodgkins and L. Bergström, Chem. Mater., 2006, 18, 4933-4938.

13 F. Akhtar, A. Ojuva, S. K. Wirawan, J. Hedlund and L. Bergström, J. Mater. Chem., 2011, 21, 8822-8828.

14 H. Cölfen and A. Völkel, Prog. Colloid Polym. Sci., 2006, 131, 126-128.

15 P. Bots, L. G. Benning, J.-D. Rodriguez-Blanco, T. RoncalHerrero and S. Shaw, Cryst. Growth Des., 2012, 12, 3806-3814.

16 T. Ogino, T. Suzuki and K. Sawada, Geochim. Cosmochim. Acta, 1987, 51, 2757-2767.

17 J. L. Wray and F. Daniels, J. Am. Chem. Soc., 1957, 79, 2031-2034.

18 J. D. Rodriguez-Blanco, S. Shaw and L. G. Benning, Nanoscale, 2011, 3, 265-271. 
19 L. Brečević and A. E. Nielsen, J. Cryst. Growth, 1989, 98, 504-510.

20 J. R. Clarkson, T. J. Price and C. J. Adams, J. Chem. Soc., Faraday Trans., 1992, 88, 243-249.

21 H. S. Lee, T. H. Ha and K. Kim, Mater. Chem. Phys., 2005, 93, 376-382.

22 D. Gebauer, P. N. Gunawidjaja, J. Y. P. Ko, Z. Bacsik, B. Aziz, L. Liu, Y. Hu, L. Bergström, C.-W. Tai, T.-K. Sham, M. Edén and N. Hedin, Angew. Chem. Int. Ed., 2010, 49, 8889-8891.

23 D. Gebauer, V. Oliynyk, M. Salajkova, J. Sort, Q. Zhou, L. Bergström and G. Salazar-Alvarez, Nanoscale, 2011, 3, 3563-3566.

24 Z. Zhao, V. Buscaglia, M. Viviani, M. T. Buscaglia, L. Mitoseriu, A. Testino, M. Nygren, M. Johnsson and P. Nanni, Phys. Rev. B: Condens. Matter, 2004, 70, 0241071-024107-8.

25 X. Xu, A. Cai, R. Liu, H. Pan, R. Tang and K. Cho, J. Cryst. Growth, 2008, 310, 3779-3787.

26 G. Cai, G. Zhao, X. Wang and S.-H. Yu, J. Phys. Chem. C, 2010, 114, 12948-12954.

27 M. Faatz, F. Gröhn and G. Wegner, Adv. Mater., 2004, 16, 996-1000.

28 Y. Kojima, M. Kanai and N. Nishimiya, Ultrason. Sonochem., 2012, 19, 325-329.

29 A. V. Radha, T. Z. Forbes, C. E. Killian, P. U. P. A. Gilbert and A. Navrotsky, Proc. Natl. Acad. Sci. U. S. A., 2010, 107, 16438-16443.
30 X. Xu, J. T. Han, D. W. Kim and K. Cho, J. Phys. Chem. B, 2006, 110, 2764-2770.

31 F. H. Chung, J. Appl. Crystallogr., 1974, 7, 519-525.

32 J. Bolze, B. Peng, N. Dingenouts, P. Panine, T. Narayanan and M. Ballauff, Langmuir, 2002, 18, 8364-8369.

33 J. Jamieson, J. Chem. Phys., 1953, 21, 1385-1390.

34 J. Kawano, N. Shimobayashi, A. Miyake and M. Kitamura, J. Phys.: Condens. Matter, 2009, 21, 425102.

35 G.-T. Zhou, Q.-Z. Yao, J. Ni and G. Jin, Am. Mineral., 2009, 94, 293-302.

36 T. Yoshino, K. Maruyama, H. Kagi, M. Nara and J. C. Kim, Cryst. Growth Des., 2012, 12, 3357-3361.

37 F. Baitalow, G. Wolf and H.-G. Schmidt, J. Therm. Anal. Calorim., 1998, 52, 5-16.

38 J. Perić, M. Vučak, R. Krstulović, L. Brečević and D. Kralj, Thermochim. Acta, 1996, 277, 175-186.

39 G. Wolf, E. Königsberger, H. G. Schmidt, L.C. Königsberger and H. Gamsjäger, J. Therm. Anal. Calorim., 2000, 60, 463-472.

40 G. Wolf, J. Lerchner, H. Schmidt, H. Gamsjäger, E. Königsberger and P. Schmidt, J. Therm. Anal., 1996, 46, 353-359.

41 J. Ihli, Y.-Y. Kim, E. H. Noel and F. C. Meldrum, Adv. Funct. Mater., 2012, DOI: 10.1002/adfm.201201805.

42 C. J. Stephens, S. F. Ladden, F. C. Meldrum and H. K. Christenson, Adv. Funct. Mater., 2010, 20, 2108-2115.

43 F. Nudelman, E. Sonmezler, P. H. H. Bomans, G. de With and N. A. J. M. Sommerdijk, Nanoscale, 2010, 2, 2436-2439. 